\title{
Evaluation of the surface and the core cultures of adenoids in patients with recurrent adenotonsillitis, obstructive adenoid hypertrophy, and otitis media with effusion
}

\author{
Mehmet Karataş ${ }^{1}$, Sadık Akgün ${ }^{2}$, Sedat Doğan1, Mehmet Turğut ${ }^{3}$, Nazif Çalış ${ }^{4}$ \\ ${ }^{1}$ Department of Otorhinolaryngology, Adlyaman University Medical School, Adiyaman, Turkey \\ ${ }^{2}$ Department of Medical Microbiology, Adiyaman University Medical School, Adlyaman, Turkey \\ ${ }^{3}$ Department of Pediatrics, Adiyaman University Medical School, Adiyaman, Turkey \\ ${ }^{4}$ Department of Economy, Faculty of Business and Management Sciences, Iskenderun Technical University, Iskenderun, Turkey
}

Received: 2020-11-07

Accepted: $2020-12-27$

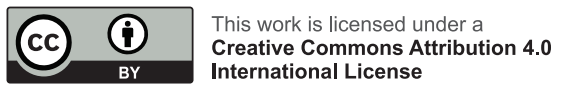

J Clin Med Kaz 2021; 18(1):38-43

Corresponding author:

Sadık Akgün.

E-mail: sakgungizem $@$ hotmail.com;

ORCID: 0000-0002-1413-0450

\section{Abstract}

Objective: To determine and to compare the core and the surface bacteriologies of the adenoids of the pediatric patients with obstructive adenoid hypertrophy, recurrent adenotonsillitis, and otitis media with effusion and to evaluate antibiotic susceptibility test patterns of the potential pathogen bacteria.

Material and methods: This prospective study was performed on 230 pediatric patients operated for either OAH, RAT, and OME during the time period from April 2014 to July 2017 in the Otorhinolaryngology Clinic of Adıyaman University Education and Research Hospital.

Results: The potential pathogen bacteria isolated from the adenoids' surfaces and cores of obstructive adenoid hypertrophy, recurrent adenotonsillitis, and otitis media with effusion groups were as follows: $33 / 148$ (22.3\%)-17/148 (11.5\%), 9/61 (14.8\%)-5/61 (8.2\%), and 6/21 (28.6\%)-3/21 (14.3\%) (p>0.05).

Conclusion: There was not statistical difference between the adenoids' surface and core cultures of obstructive adenoid hypertrophy, recurrent adenotonsillitis, and otitis media with effusion.

Key words: throat infection, adenotonsillitis, throat culture, antibiotic sensitivity test

\section{Introduction}

The nasopharyngeal tonsil or adenoid is located at the junction of the roof and posterior wall of the nasopharynx. Adenoid together with the tubal, palatine, and lingual tonsils, and lateral pharyngeal bands constitute the transition zone between respiratory and alimentary tracts which is called as Waldeyer's ring (WR), or nasal-associated lymphoid tissue (NALT) [1]. It is an effector organ in both mucosal-type and systemic-type adaptive immunity and possesses an important role in immune protection of upper respiratory tract (URT) [2]. Due to its location and immunologic function, adenoid has been implied in the pathogenesis of various infectious and noninfectious processes of URT [3]. Children with infections associated with adenoid are referred to both pediatric and otolaryngology clinics and they are treated with multiple courses of antibiotics before surgery. However, many of them reveal persistence of pathogenic bacteria in their nasopharynx because adenoid tissue acts as a reservoir [4]. Adenoid may cause Eustachian dysfunction/middle ear infections or effusions [5,6], rhinosinusitis, adenotonsillitis, obstructive sleep apnea, facial growth abnormalities, swallowing problems, smell and taste disorders, and speech problems due to either being a reservoir for bacteria or its obstructive effect $[7,8]$. Adenoid hypertrophy (AH) is a commonly encountered obstructive adenoid pathology and patients with $\mathrm{AH}$ mostly presents with snoring, otitis media, cessation of 
respiration, chronic mouth breathing [9]. Adenoidectomy alone is the treatment of choice for both reservoir-associated and obstructive pathologies of adenoids. Recurrent adenotonsillitis-3 per year for 3 years, 5 per year for 2 years, 7 or more in 1year- (RAT) is also one of the most commonly seen diseases of the childhood and antibiotic therapy is first line treatment. Since antibiotherapy is usually insufficient, adenoidectomy is performed in conjunction with tonsillectomy [10]. Otitis media with effusion (OME) is mostly seen between ages of 6 months and 4 years, characterised with the presence of middle ear fluid without signs of infection or inflammation, and may occur during an URT infection (URTI) or an inflammatory response following acute otitis media [11]. The preferred treatment approach is adenoidectomy together with ventilation tube insertion to tympanic membrane in patients with OME for at least three months.

This study was carried out to determine and to compare the core and the surface bacteriologies in the adenoids of the pediatric patients with obstructive adenoid hypertrophy $(\mathrm{OAH})$, recurrent adenotonsillitis (RAT), and OME and to evaluate antibiotic susceptibility test patterns.

\section{Material and methods Patients and microbiological methods}

The present study was designed prospectively and performed on 230 pediatric patients operated for either OAH, RAT, and OME during the time period from April 2014 to July 2017 in the Otorhinolaryngology Clinic of Adiyaman University Education and Research Hospital (Adiyaman, Turkey). They were divided into three groups according to diagnosis: $\mathrm{OAH}$ group (adenoidectomy only), RAT group (adenotonsillectomy), and OME group (adenoidectomy together with ventilation tube insertion to tympanic membrane). Furthermore, bacteriologic examinations of adenoids in all three groups, tonsils in RAT group, and middle ear aspirates in OME group were done by Microbiology Department of the same hospital. Inclusion criteria for the study were as follows: age between 2 and 13 years, endoscopic or radiologic diagnosis of nasopharyngeal obstruction by adenoid in OAH group, history of recurrent adenotonsillitis in RAT group, and persistence of middle ear effusion for at least 3 months in OME group. Exclusion criteria were as follows; antibiotic use up to 1 month prior to the procedure, cleft palate, allergy, and presence of any acute infection.

While the samples with non-pathogen bacteria and normal oro- and nasopharyngeal flora including coagulase-negative staphylococci (CNS) (S. epidermidis, S. haemolyticus, S. saprophyticus, S. lugdunensis,S. hominis), alpha-hemolytic streptococci (AHS) (viridans streptococci including S. mitis, S. mitior, S. sanguis, S. mutans, S. anginosus), non-hemolytics treptococci (NHS) (Enterococcus faecalis, Enterococcus faecium), Corynebacterium spp., Neisseria spp., Prevotella spp., and Peptostreptococci were regarded as normal flora (NF), the ones including S. aureus, Group A beta-hemolytic streptococci (GABHS) (S. pyogenes), alpha-hemolytic streptococci other than viridans (S. pneumoniae), H. influenzae, E. coli, P. aeruginosa, and $\mathrm{M}$. catarrhalis were considered as the potential pathogenic bacteria (PPB). Therefore, after antibiotic-susceptibility testing, antibiotic efficacy (AE) for each antibiotic used in sensitivity determinations was established on the basis of the percent sensitivity of a given organism to the given antibiotic [12].

a) Sample acquisition and transfer: All operations were carried out under general anestesia with orotracheal intubation.
After application of an oral retainer and retraction of the uvula and soft palate, the adenoid tissue was visualized well and then adenoid surface swabs were obtained with a sterile cottontipped applicator without oral contamination. Just after the removal of adenoid tissue with a Beckmann adenotome curette and irrigation with sterile saline solution, the deep surface of the adenoid was exposed with a sterile scalpel and was swabbed with the applicator (Figure 1).

However, in addition to the aforementioned common steps, middle ear fluid was swabbed too in OME group. Furthermore, all swabbing samples from core, surface, and middle ear fluid were performed twice (one sample for aerobes and one for anaerobes) at the same time in all three groups. All swab specimens were

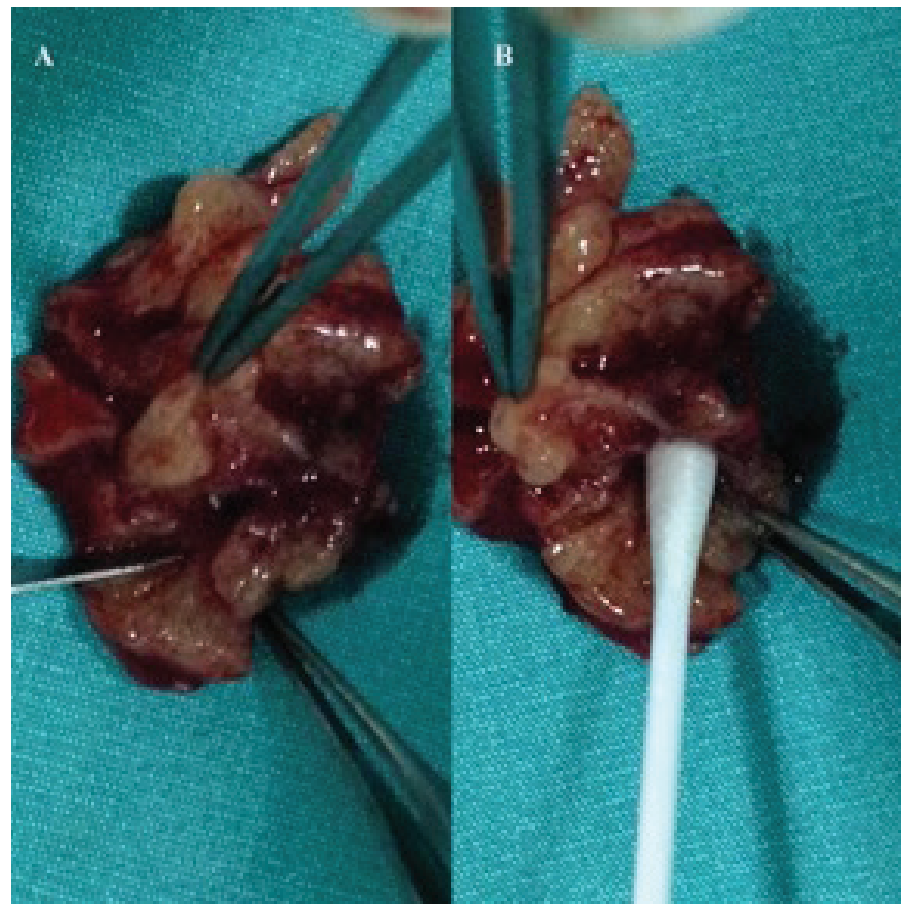

Figure 1. Acquisition of adenoid core culture: exposition of the deep surface of the adenoid with a sterile scalpel (A) and swabbing the core with the applicator (B).

placed in Stuart's transport medium (Gül Ka, Ivogsan, Ankara, Türkiye) and transported to the microbiological laboratory within $15 \mathrm{~min}$.

b) Inoculation and incubation of samples: Both aerobic and anaerobic samples were inoculated onto 5\% Sheep Blood Agar (SBA), Eosine Methylene Blue Agar (EMBA), and Chocolate Agar (CA) plates (Becton Dickinson, USA). Anaerobic conditions were achieved by using anaerobic jars and kits (GasPak EZ Anaerobe Pouch; Becton Dickinson). The samples were kept in a bacteriological incubator (Nüve Incubator EN 120, Ankara, Turkey) for18 to 24 hours.

c) Evaluation of bacterial growth: The day after the inoculation, the bacterial colonies grown on plateswere subjected to preliminary identification tests using conventional methods (Catalase, Coagulase, Oxidase, Gram Staining). After discrimination of Gram negative and Gram positive bacteria was done, the plates suspected to be pathogenic were eliminated.

d) Identification and Susceptibility Testing: For bacterial identification and antibiotic susceptibility testing, the suspected pathogenic plates were finally tested using an automated identification and susceptibility testing system (BD Phoenix 100, Becton Dickinson, USA) using appropriate kits (GP ID/AST, GN ID/AST, S ID/AST, Becton Dickinson, USA). Antibiotic susceptibility of each PPB was tested with 
27 antibiotics including Penicillin G, Ampicillin, Trimetoprimsulfametoxazole, Cefazolin, Clindamycine, Tetracycline, Amikacin, Ciprofloxacin, Gentamycine, Cefuroxime, Vancomycin, Teicoplanin, Amoxicillin-Clavulanic acid, Ofloxacin, Imipenem, Sulbactam-Ampicillin, Meropenem, Oxacillin, Linezolid, Levofloxacin, Cefoxitine, Ertapenen, Fosfomisin, Nitrofurantoin, Cefoperazon-sulbactam, Ceftazidime, and Tazobactam+Piperacillin.

\section{Ethical approval}

The Institutional Ethical Committee of Medical Faculty of Adiyaman University approved this study (2014/04-5) and informed consent was obtained from all of children's parents for both surgery and donating the tissue samples for the study.

\section{Statistical analysis}

Categorical variables were expressed as numbers and percentages. Mean, standard deviation, and minimum and maximum values were used for the descriptive statistics. The $\mathrm{Z}$ score for two population proportions was performed for the comparison of observed distribution in different groups and antibiotic-susceptibilities. The $\mathrm{P}$ value was equal to 0.05 .

\section{Results}

Of the 230 children, 97 were females (42\%), 133 were males $(58 \%)$. Their age ranged from 2 to 13 years (mean: $6.68 \pm 2.36)$. Of these, 148 underwent adenoidectomy only $(\mathrm{OAH}$ group), 61 operated for adenotonsillectomy (RAT group), and 21 had adenoidectomy with tympanostomy tube placement (OME group).

The PPB isolated from the adenoids' surfaces and cores of OAH, RAT, and OME groups were as follows: 33/148 (22.3\%)$17 / 148(11.5 \%), 9 / 61(14.8 \%)-5 / 61(8.2 \%)$, and 6/21 (28.6\%)$3 / 21(14.3 \%)(p>0.05)$. Therefore, the overall isolation of the PPB from the either adenoid surface or core specimens of OAH, RAT, and OME groups was 34/148 (23\%), 10/61 (16.4\%), and 7/21 $(33.3 \%)$ with an overall average of $51 / 230(22.2 \%)$, respectively $(\mathrm{P}>0.05)$. S. aureus was the most commonly cultured PPB in either surface or core cultures of OAH, the RAT, and the OME groups $(23(15.5 \%), 9(14.8 \%)$, and $2(9.5 \%))$ with an overall average of $34 / 230(14.8 \%)(p>0.05)$. S. pneumonia was also seen in all three groups (OAH-3/148 (2\%), RAT-1/61 (1.6\%), and OME-1/21 (4.7\%)). E. coli could be cultured only in the cores of both the OAH (one patient) and the OME (one patient) groups but not in the surfaces. P. aeruginosa were seen in the OAH ( 3 patients) and the OME (3 patients) groups but could not be cultured in the RAT group. GABHS-S. pyogenes was only obtained in the OAH group (4/148 (2.7\%)). Neither H. influenza nor M. catarrhalis was not grown on any plate. While 7 patients revealed no growth, 70 patients had normal flora bacteria in both surface and core cultures. 102 patients showed normal flora and no growth. The detailed data about each PPB obtained from the adenoids' surfaces and cores of OAH, RAT, and OME groups are tabled in Table 1 and 2.

Pathogenic microflora isolated from the adenoids of all three groups.

\begin{tabular}{|c|c|c|c|c|c|c|c|c|c|}
\hline \multirow[b]{2}{*}{$\begin{array}{l}\text { Pathogen } \\
\text { bacteria }\end{array}$} & \multicolumn{3}{|l|}{ OAH n=148 } & \multicolumn{3}{|l|}{ RAT $n=61$} & \multicolumn{3}{|l|}{$\mathrm{EOM} n=21$} \\
\hline & $\begin{array}{l}\text { Surface } \\
\text { culture (n) }\end{array}$ & $\begin{array}{l}\text { Core culture } \\
\text { (n) }\end{array}$ & $\begin{array}{l}\text { Surface and } \\
\text { Core culture } \\
\text { (n) }\end{array}$ & $\begin{array}{l}\text { Surface } \\
\text { culture (n) }\end{array}$ & $\begin{array}{l}\text { Core culture } \\
\text { (n) }\end{array}$ & $\begin{array}{l}\text { Surface and } \\
\text { Core culture } \\
\text { (n) }\end{array}$ & $\begin{array}{l}\text { Surface } \\
\text { culture (n) }\end{array}$ & $\begin{array}{l}\text { Core culture } \\
\text { (n) }\end{array}$ & \begin{tabular}{|l} 
Surface \\
and Core \\
culture (n)
\end{tabular} \\
\hline S. aureus & 23 & 12 & 12 & 8 & 4 & 3 & 2 & 2 & 2 \\
\hline $\begin{array}{l}\text { GABHS (S. } \\
\text { pyogenes) }\end{array}$ & 4 & 2 & 2 & 0 & 0 & 0 & 0 & 0 & 0 \\
\hline $\begin{array}{l}\text { AHS S. } \\
\text { pneumonia) }\end{array}$ & 3 & 0 & 0 & 1 & 1 & 1 & 1 & 0 & 0 \\
\hline H. influenzae & 0 & 0 & 0 & 0 & 0 & 0 & 0 & 0 & 0 \\
\hline E. coli & 0 & 1 & 0 & 0 & 0 & 0 & 0 & 1 & 0 \\
\hline P. aeruginosa & 3 & 2 & 2 & 0 & 0 & 0 & 3 & 0 & 0 \\
\hline M. catarrhalis & 0 & 0 & 0 & 0 & 0 & 0 & 0 & 0 & 0 \\
\hline
\end{tabular}

Table 2

Pathogenic microorganisms cultured from the surface and /or core specimens of adenoids in all three groups.

\begin{tabular}{|c|c|c|c|c|}
\hline Pathogen & $\begin{array}{l}\text { OAH } \\
n=148(\%)\end{array}$ & $\begin{array}{l}\text { RAT } \\
n=61(\%)\end{array}$ & $\begin{array}{l}\text { EOM } \\
n=21(\%)\end{array}$ & $\begin{array}{l}\text { Total } \\
n=230(\%)\end{array}$ \\
\hline S. aureus & $23(15.5 \%)$ & $9(14.8 \%)$ & $2(9.5 \%)$ & $34(14.8 \%)$ \\
\hline $\begin{array}{l}\text { GABHS } \\
\text { (S. pyogenes) }\end{array}$ & $4(2.7 \%)$ & 0 & 0 & $4(1.7 \%)$ \\
\hline $\begin{array}{l}\text { AHS } \\
\text { (S. pneumonia) }\end{array}$ & $3(2 \%)$ & $1(1.6 \%)$ & $1(4.7 \%)$ & $5(2.1 \%)$ \\
\hline H. influenzae & 0 & 0 & 0 & 0 \\
\hline E. coli & $1(0.7 \%)$ & 0 & $1(4.7 \%)$ & $2(0.8 \%)$ \\
\hline P. aeruginosa & $3(2 \%)$ & 0 & $3(14.3 \%)$ & $6(2.6 \%)$ \\
\hline M. catarrhalis & 0 & 0 & 0 & 0 \\
\hline Total & $34(23 \%)$ & $10(16.4 \%)$ & 7 (33.3\%) & $51(22.2 \%)$ \\
\hline
\end{tabular}

Furthermore, the surface/core culture results of adenoids were termed as "Normal flora (NF)", "No growth (NG)", and
"Pathogen present (PP)" and the culture results of each group were analyzed in terms of these findings in Table 3. 


\begin{tabular}{|l|l|l|l|l|l|}
\hline Surface culture & Core culture & OAH n=148 & RAT $n=61$ & EOM $n=21$ & P value \\
\hline Normal flora & Normal flora & $49 / 148$ & $13 / 61$ & $8 / 21$ & $\mathrm{p}>0,05$ \\
\hline Normal flora & No growth & $57 / 148$ & $37 / 61$ & $6 / 21$ & $\mathrm{p}<0,05$ \\
\hline Normal flora & Pathogen present & $1 / 148$ & $1 / 61$ & $2 / 21$ & $\mathrm{p}<0,05$ \\
\hline Pathogen present & Normal flora & $2 / 148$ & $2 / 61$ & $1 / 21$ & $\mathrm{p}>0,05$ \\
\hline Pathogen present & No growth & $16 / 148$ & $4 / 61$ & $2 / 21$ & $\mathrm{p}>0,05$ \\
\hline Pathogen present & Pathogen present & $15 / 148$ & $3 / 61$ & $2 / 21$ & $\mathrm{p}>0,05$ \\
\hline No growth & Normal flora & $2 / 148$ & $0 / 61$ & $0 / 21$ & $*$ \\
\hline No growth & No growth & $6 / 148$ & $1 / 61$ & $0 / 21$ & $\mathrm{p}>0,05$ \\
\hline No growth & Pathogen present & $0 / 148$ & $0 / 61$ & $0 / 21$ & $*$ \\
\hline
\end{tabular}

* Statistical analysis could not be done due to a small number of subjects

Table 4

Antibiotic-susceptibility results for all potential pathogenic bacteria.

\begin{tabular}{|c|c|c|c|c|c|c|c|c|c|c|c|c|}
\hline \multirow{2}{*}{$\begin{array}{l}\text { Pathogen } \\
\text { Antibiotic*,** }\end{array}$} & \multicolumn{2}{|c|}{ S.aureus } & \multicolumn{2}{|c|}{ P. aeruginosa } & \multicolumn{2}{|c|}{ S.pneumonia } & \multicolumn{2}{|c|}{ S. pyogenes } & \multicolumn{2}{|c|}{ E. coli } & \multicolumn{2}{|c|}{ Overall } \\
\hline & $\mathrm{R}$ & $S$ & $\mathrm{R}$ & S & $\mathrm{R}$ & S & $\mathrm{R}$ & S & $\mathrm{R}$ & S & $\mathrm{R}$ & S \\
\hline PNGa & 34 & 0 & 6 & 0 & 0 & 5 & 0 & 4 & 2 & 0 & 42 & 9 \\
\hline $\mathrm{AMPb}$ & 26 & 8 & 6 & 0 & 0 & 5 & 0 & 4 & 0 & 2 & 32 & 19 \\
\hline SXTc & 0 & 34 & 6 & 0 & 5 & 0 & 4 & 0 & 0 & 2 & 15 & 36 \\
\hline TETc & 0 & 34 & 6 & 0 & 5 & 0 & 0 & 4 & 2 & 0 & 13 & 38 \\
\hline FOXc & 2 & 32 & 6 & 0 & 0 & 5 & 0 & 4 & 2 & 0 & 10 & 41 \\
\hline VAc & 0 & 34 & 6 & 0 & 0 & 5 & 0 & 4 & 2 & 0 & 8 & 43 \\
\hline NITC & 0 & 34 & 6 & 0 & 0 & 5 & 0 & 4 & 2 & 0 & 8 & 43 \\
\hline $\mathrm{CZc}$ & 2 & 32 & 6 & 0 & 0 & 5 & 0 & 4 & 0 & 2 & 8 & 43 \\
\hline LNZc & 0 & 34 & 6 & 0 & 0 & 5 & 0 & 4 & 2 & 0 & 8 & 43 \\
\hline DAc & 0 & 34 & 6 & 0 & 0 & 5 & 0 & 4 & 2 & 0 & 8 & 43 \\
\hline TECc & 0 & 34 & 6 & 0 & 0 & 5 & 0 & 4 & 1 & 1 & 7 & 44 \\
\hline AMCd & 0 & 34 & 6 & 0 & 0 & 5 & 0 & 4 & 0 & 2 & 6 & 45 \\
\hline SAMd & 0 & 34 & 5 & 1 & 0 & 5 & 0 & 4 & 0 & 2 & 5 & 46 \\
\hline MEMd & 0 & 34 & 0 & 6 & 5 & 0 & 0 & 4 & 0 & 2 & 5 & 46 \\
\hline OFXd & 0 & 34 & 1 & 5 & 0 & 5 & 0 & 4 & 2 & 0 & 3 & 48 \\
\hline OXd & 0 & 34 & 1 & 5 & 0 & 5 & 0 & 4 & 2 & 0 & 3 & 48 \\
\hline FFd & 0 & 34 & 2 & 4 & 0 & 5 & 0 & 4 & 0 & 2 & 2 & 49 \\
\hline ETPd & 0 & 34 & 2 & 4 & 0 & 5 & 0 & 4 & 0 & 2 & 2 & 49 \\
\hline LEVd & 0 & 34 & 1 & 5 & 0 & 5 & 0 & 4 & 0 & 2 & 1 & 50 \\
\hline CXMd & 0 & 34 & 1 & 5 & 0 & 5 & 0 & 4 & 0 & 2 & 1 & 50 \\
\hline CAZd & 0 & 34 & 0 & 6 & 0 & 5 & 0 & 4 & 1 & 1 & 1 & 50 \\
\hline AKd & 0 & 34 & 0 & 6 & 0 & 5 & 0 & 4 & 0 & 2 & 0 & 51 \\
\hline CIPd & 0 & 34 & 0 & 6 & 0 & 5 & 0 & 4 & 0 & 2 & 0 & 51 \\
\hline GNd & 0 & 34 & 0 & 6 & 0 & 5 & 0 & 4 & 0 & 2 & 0 & 51 \\
\hline IMPd & 0 & 34 & 0 & 6 & 0 & 5 & 0 & 4 & 0 & 2 & 0 & 51 \\
\hline SCPd & 0 & 34 & 0 & 6 & 0 & 5 & 0 & 4 & 2 & 0 & 0 & 51 \\
\hline TZPd & 0 & 34 & 0 & 6 & 0 & 5 & 0 & 4 & 0 & 2 & 0 & 51 \\
\hline
\end{tabular}

R: Resistant, S: Sensitive

* Penicillin G (PNG), Ampicillin (AMP), Trimetoprim-sulfametoxazole (SXT), Cefazolin (CZ), Clindamycine (DA), Tetracycline (TET), Amikacin (AK), Ciprofloxacin (CIP), Gentamycine (GN), Cefuroxime (CXM), Vancomycin (VA), Teicoplanin (TEC), Amoxicillin-Clavulanic acid (AMC), Ofloxacin (OFX), Imipenem (IMP), Sulbactam-Ampicillin (SAM), Meropenem (MEM), Oxacillin (OX), Linezolid (LNZ), Levofloxacin (LEV), Cefoxitine (FOX), Ertapenen (ETP), Fosfomisin (FF), Nitrofurantoin (NIT), Cefoperazon-sulbactam (SCP), Ceftazidime (CAZ), and Tazobactam+Piperacillin (TZP).

** Antibiotic efficacy of each antibiotic; a Class A (17.6\%), b Class B (37.25\%), c Class C (70.58\%-86.27\%), and d class D (88.23-100\%).

The antibiotics were divided into four classes in terms of $\mathrm{AE}$ as follows: class A (17.6\%), class B (37.25\%), class C (70.58\%-86.27\%), and class D (88.23-100\%) and antibioticsusceptibility results for all PPB were indicated in Table 4.

\section{Discussion}

Nasopharynx harbours the adenoid which continuously exposes to environmental allergens and microorganisms during respiration with resultant $\mathrm{AH}$ achieving peak size at 3 to 7 years of age and then regressing $[13,14]$. The clinical importance of adenoid does not become apparent until adenoid leads to nasal airway or eustachian tube obstruction. While OAH presents with triad of snoring, daytime and nighttime mouthbreathing, and hyponasal speech, RAT reveals several episodes of adenotonsillitis, and OME usually manifests with conductive hearing loss. There are many studies in the literature describing adenoid microbiology $[8,15,16]$. Brook et al. compared the bacteriology of a healthy adenoid group with 3 diseased adenoid 
groups including recurrent otitis media, OAH, and RAT ones using quantitative bacteriological method and found that that the bacterial load of the adenoids of children with ROM, RAT, and $\mathrm{OAH}$ is higher than in normal adenoids [17]. Rajeshwary et al. assessed the bacteriology of the adenoid tissue in chronic adenotonsillitis and $\mathrm{AH}$, and the antibiotic sensitivity of PPBs and concluded that with appropriate antibiotic treatment of adenoid hypertrophy will prevent unnecessary exposure to repeated antimicrobials with resultant maintenance of the beneficial effects of the normal adenoid flora and thereby colonization of the adenoid by potential pathogens will be inhibited [4]. Almaç et al. obtained superficial and deep swab cultures from hypertrophied adenoids of children with or without OME, found no statistical difference between deep and surface PPB of both groups and the low colonization levels of middle ear PPB in adenoid tissue, and deduced that the colonization of potential pathogens of middle ear in adenoid tissue may not be significant factor for the etiopathogenesis of OME [3]. Feket-Szabo et al. compared the bacterial cultures of nasopharyngeal swabs and deep parts of adenoidectomy samples of 20 children with recurrent upper respiratory tract infection, and breathing difficulties due to nasal obstruction and determined the similar culture results including beside polymicrobial aerobic-anaerobic flora in all patients, predominant aerobic isolates of $\mathrm{S}$. pneumoniae, $\mathrm{H}$. influenzae and M. catarrhalis and anaerobic ones of Peptostreptococcus spp., Prevotella spp., and Fusobacterium spp. were obtained in both groups [18]. Daniel et al. examined bacterial involvement in OME by using a bacterial viability stain and confocal laser scanning microscopy and demonstrated live bacteria in 57/62 (91.8\%) which strongly suggests that bacteria and biofilms are of importance in the aetiopathogenesis of OME [19]. Taylan et al. investigated the bacterial flora of core and surface microorganisms and pathogen microorganisms' beta-lactamase production rate of 91 children undergoing adenotonsillectomy for RAT and/or obstructive symptoms, demonstrated that both adenoid and tonsil tissues possessed polymicrobial aerobicanaerobic flora and similar bacteriology, and suggested that treatment failures of tonsillitis may be due to S. aureus and other beta-lactamase-producing bacteria [10]. Gul et al. evaluated tonsillar surface swab and deep tissue cultures of 116 patients with RAT, found PPB in 77 patients of which 25 had same pathogens and 52 revealed different types of pathogens from the surface and deep cultures, detected surface cultures pathogens as, in descending frequency, GABHS, S. pneumoniae, S. aureus, and $\mathrm{H}$. influenza, and stated that since the real pathogens of the tonsils may not always be obtained from the tonsillar surface swab, besides the target pathogens, antibiotic coverage also for H. influenza may be chosen [20].

PPB was cultured in either adenoids' surface and/or core of 51 children. There was no statistical difference between the number of PPB isolated from the adenoids' surfaces and cores of OAH, RAT, and OME groups. Therefore, the overall ratio of the surface with PPB to the core with PPB was 2, thus just half of PPB-bearing adenoid surfaces revealed positive core cultures for PPB. Thus, core culture of adenoid may not reveal the same PPB with surface culture. The overall isolation of the PPB from the either adenoid surface or core specimens of OAH, RAT, and OME groups did not reveal statistical difference. The remaining of the specimens revealed either normal flora or no growth. However, isolation of any microorganism (neither PPB nor NF) could not be achieved in the middle ear cultures of the OME group. S. aureus was the most commonly cultured PPB (34) in either surface or core cultures of OAH, the RAT, and the OME groups with no statistically significant difference between the groups. P. aeruginosa, S. pneumonia, S. pyogenes, and E. coli, and were found in small amounts $(6 / 230,5 / 230,4 / 230$, and $2 / 230$, respectively), while neither $H$. influenza nor $M$. catarrhalis was not grown on any plate.

AE of each antibiotic for PPB represented that Penicillin $\mathrm{G}$ and Ampicillin are least effective antibiotics against PPB of adenoids while class D antibiotics are most effective.

H. influenza type $b$ vaccine (Hib) and the 7-valent pneumococcal conjugated vaccine (PCV7) were introduced into the National Immunization Program of Turkey in 2007 and 2008, respectively and PCV7 was switched to the 13-valent pneumococcal conjugated vaccine (PCV13) in 2011 [21,22]. This may explain why $\mathrm{H}$. influenza could not be cultured on any plates and S. pneumonia was cultured in small amounts when compared with aforementioned studies.

\section{Conclusion}

There was not statistical difference between the adenoids' surface and core cultures of OAH, RAT, and OME. However, the introduction of $\mathrm{H}$. influenza type $\mathrm{b}$ vaccine and pneumococcal conjugated vaccine into the National Immunization Program of Turkey has conducted to realize the effect of the vaccines on the nasopharyngeal colonization by $\mathrm{H}$. influenza and $\mathrm{S}$. pneumonia which are primary pathogens implied in many otolaryngologic infectious diseases. Furthermore, the choice of antibiotics for nasopharyngeal infections in pediatric patients should be performed meticulously according to the antibioticsusceptibility tests and the antibiotic efficacy findings.

Disclosures: There is no conflict of interest for all authors.

Acknowledgements: We thank to Adryaman Training and Research Hospital, Medical Microbiology culture laboratory staff for their contributions.

Funding: The research received no specific grant from any funding agency in the public, commercial, or not-for-profit sectors.

\section{References}

1. Hellings P, Jorissen M, Ceuppens JL. The Waldeyer's ring. Acta Oto-rhino-laryngologica Belgica. 2000; 54(3):237-241. PMID: 11082757

2. Brandtzaeg P. Immunology of tonsils and adenoids: everything the ENT surgeon needs to know. Int J Pediatr Otorhinolaryngol. 2003; 67(1):69-76. https://doi.org/10.1016/j.ijporl.2003.08.018

3. Almac A, Elicora SS, Yumuk Z, Dundar V, Willke A. The relationship between chronic otitis media with effusion and surface and deep flora of hypertrophic adenoids. Int J Pediatr Otorhinolaryngol. 2009; 73(10):1438-1440. https://doi.org/10.1016/j.ijporl.2009.07.014

4. Rajeshwary A, Rai S, Somayaji G, Pai V. Bacteriology of symptomatic adenoids in children. N Am J Med Sci. 2013; 5(2):113-8. https:// doi.org/10.4103/1947-2714.107529 
5. Suzuki M, Watanabe T, Mogi G. Clinical, bacteriological and histological study of adenoids in children. Am. J. Otolaryngol. 1999; 20(2):85-90. https://doi.org/10.1016/S0196-0709(99)90016-9

6. Bernstein JM. Waldeyer's ring and otitis media: the nasopharyngeal tonsil and otitis media. Int J Pediatr Otorhinolaryngol. 1999; 49(1):127-32. https://doi.org/10.1016/S0165-5876(99)00146-9

7. Marseglia GL, Caimmi D, Pagella F, Matti E, Labó E, Licari A, Salpietro A, Pelizzo G, Castellazzi AM. Adenoids during childhood: the facts. Int J Immunopathol Pharmacol. 2011; 24(41):1-5. doi: 10.1177/03946320110240S401

8. Brook I. Aerobic and anaerobic bacteriology of adenoids in children: a comparison between patients with chronic adenotonsillitis and adenoid hypertrophy. Laryngoscope. 1981; 91(3):377-82. https://doi.org/10.1288/00005537-198103000-00005

9. Saedi B, Sadeghi M, Mojtahed M, Mahboubi H. Diagnostic efficacy of different methods in the assessment of adenoid hypertrophy. Am J Otolaryngol. 2011; 32(2):147-51. doi: 10.1016/j.amjoto.2009.11.003

10. Taylan I, Ozcan I, Mumcuoğlu I, Baran I, Murat Özcan K, Akdoğan O, Selcuk A, Balaban N, Dere H. Comparison of the surface and core bacteria in tonsillar and adenoid tissue with Beta-lactamase production. Indian J Otolaryngol Head Neck Surg. 2011; 63(3):223-8. https://doi.org/10.1007/s12070-011-0265-z

11. Paradise JL, Rockette HE, Colborn DK, Bernard BS, Smith CG, Kurs-Lasky M, Janosky JE. Otitis media in 2253 Pittsburgh-area infants: prevalence and risk factors during the first two years of life. Pediatrics. 1997; 99(3):318-33. https://doi.org/10.1542/peds.99.3.318

12. Gabrielson ML, Stroh E. Antibiotic efficacy in odontogenic infections. J Oral Surg. 1975; 33(8):607-10. PMID: 1056464.

13. Modrzynski M, Zawisza E. An analysis of the incidence of adenoid hypertrophy in allergic children. Int J Pediatr Otorhinolaryngol. 2007; 71(5):713-9. https://doi.org/10.1016/j.ijporl.2006.12.018

14. Berçin AS, Ural A, Kutluhan A, Yurttaş V. Relationship between sinusitis and adenoid size in pediatric age group. Ann Otol Rhinol Laryngol. 2007; 116(7):550-3. https://doi.org/10.1177/000348940711600711

15. DeDio RM, Tom LW, McGowan KL, Wetmore RF, Handler SD, Potsic WP. Microbiology of the tonsils and adenoids in a pediatric population. Arch Otolaryngol Head Neck Surg. 1988; 114(7):763-5. doi: 10.1001/archotol.1988.01860190067025

16. Pillsbury HC 3rd, Kveton JF, Sasaki CT, Frazier W. Quantitative bacteriology in adenoid tissue. Otolaryngol Head Neck Surg. 1981; 89(3 Pt 1):355-63. https://doi.org/10.1177/019459988108900301

17. Brook I, Shah K, Jackson W. Microbiology of healthy and diseased adenoids. Laryngoscope. 2000; 110(6):994-9. https://oi. org/10.1097/00005537-200006000-00021

18. Fekete-Szabo G, Berenyi I, Gabriella K, Urban E, Nagy E. Aerobic and anaerobic bacteriology of chronic adenoid disease in children. Int J Pediatr Otorhinolaryngol. 2010; 74(11):1217-20. https://doi.org/10.1016/j.ijporl.2010.07.013

19. Daniel M, Imtiaz-Umer S, Fergie N, Birchall JP, Bayston R. Bacterial involvement in otitis media with effusion. Int J Pediatr Otorhinolaryngol. 2012; 76(10):1416-22. https://doi.org/10.1016/j.ijporl.2012.06.013

20. Gul M, Okur E, Ciragil P, Yildirim I, Aral M, Akif Kilic M. The comparison of tonsillar surface and core cultures in recurrent tonsillitis. Am J Otolaryngol. 2007; 28(3):173-6. https://doi.org/10.1016/j.amjoto.2006.08.010

21. Marijam A, Olbrecht J, Ozakay A, Eken V, Meszaros K. Cost-Effectiveness Comparison of Pneumococcal Conjugate Vaccines in Turkish Children. Value in Health Regional Issues. 2019; 19:34-44. https://doi.org/10.1016/j.vhri.2018.11.007

22. Dinleyici M, Carman KB, Kilic O, Laciner Gurlevik S, Yarar C, Dinleyici EC. The immunization status of children with chronic neurological disease and serological assessment of vaccine-preventable diseases. Hum Vaccin Immunother. 2018; 14(8):1970-1976. https://doi.org/10.1080/21645515.2018.1460986 